\title{
Implementation of the Omni Vehicle Dynamics on Modelica
}

\author{
Ivan Kosenko $^{1} \quad$ Kirill Gerasimov ${ }^{2}$ \\ ${ }^{1}$ Moscow State Technical University of Radio Engineering, Electronics, and Automation \\ Department of Engineering Mechanics \\ Vernadsky Avenue 78, 119454, Moscow, Russia \\ ${ }^{2}$ Lomonosov Moscow State University \\ Department of Theoretical Mechanics and Mechatronics \\ Leninskiye Gory 1, Main Building, GSP-1, 119991, Moscow, Russia
}

\begin{abstract}
Omni wheel is defined as one having rollers along its rim. Respectively omni vehicle is one equipped by omni wheels. Several steps of development of dynamical model of the omni vehicle multibody system are implemented. Essential parameters of the model: (a) number of rollers per the wheel, and (b) angle of the roller axis inclination to the wheel plane, are introduced. Initially, dynamics of the free roller moving in a field of gravity and having a unilateral contact constraint with horizontal surface is modeled. The contact tracking using simplified and efficient algorithm turns out being possible. On the next stage the omni wheel model is developed and debugged. After that the whole vehicle model is assembled as a container class having arrays of objects as instantiated classes / models of omni wheels and joints. Dynamical properties of the resulting model are illustrated via numerical experiments.
\end{abstract}

Keywords: omni wheel; contact tracking; unilateral constraint; contact detection; model of friction

\section{Introduction}

Investigation of omni vehicle dynamical properties is sufficiently popular topic in frame of the multibody dynamics $[1,2,3,4]$. The omni vehicle is one having omni wheels, wheels equipped by rollers along the rim. Simplified, idealized models having contacting rollers as an infinitely small discrete elements are known. Thus one has a resulting non-holonomic constraint being "uniformly distributed" over the wheel rim. As a result, paradoxically, the physical objects, omni wheels, describe approximately, in this situation, an idealized object, "simplified" infinitesimal model.

Our goal in this paper is to develop a technique for building up a dynamical prototype for the "real" model of the omni vehicle explicitly involving dynamics of physical rollers. Here we rely upon the "simple"3D multibody dynamics library classes utilized previously in several examples of the multibody systems dynamics [5]. Simultaneously this library enables us to create complex dynamical models including unilateral constraints of different nature.

Unlike to $[2,3]$ we emphasize here on the details of the unilateral constraint implementation paying special attention to contact switching when rollers changing.

\section{Problem formulation}

Upon describing the omni vehicle model construct note that the number of rollers per each wheel and the angle of inclination of the roller axis of symmetry to the wheel plane are both fundamental parameters of the vehicle dynamical model. For simplicity and presentation clarity we currently consider omni wheels being equipped by four rollers. Also, for simplicity rollers themselves have their axes of symmetry lying in the wheel plane, see Figure 1. These fundamental parameters are easily changed in a way simple enough. We assume also that the rollers are located on the omni wheel such that for wheel vertically aligned a projection of the curve of contact consists of segments in the sequence, each segment corresponding to the contact of individual roller. These segments are connected in a way such that the normal relative velocity at contact is equal to zero at the switching point of rollers. This means that the normal impact is always absent. Discontinuities of the tangent relative sliding velocity are absent for zero angle of inclination. But the tangent force of friction may have discontinuity of the first kind in the worst case of angle of inclination if the 
roller symmetry axis to the wheel plane is non-zero. Thus, the wheel linear and angular velocities will be continuous at an instant when roller switching contact. Similar statement takes place for rollers, as well. Then tangent impacts are also absent.

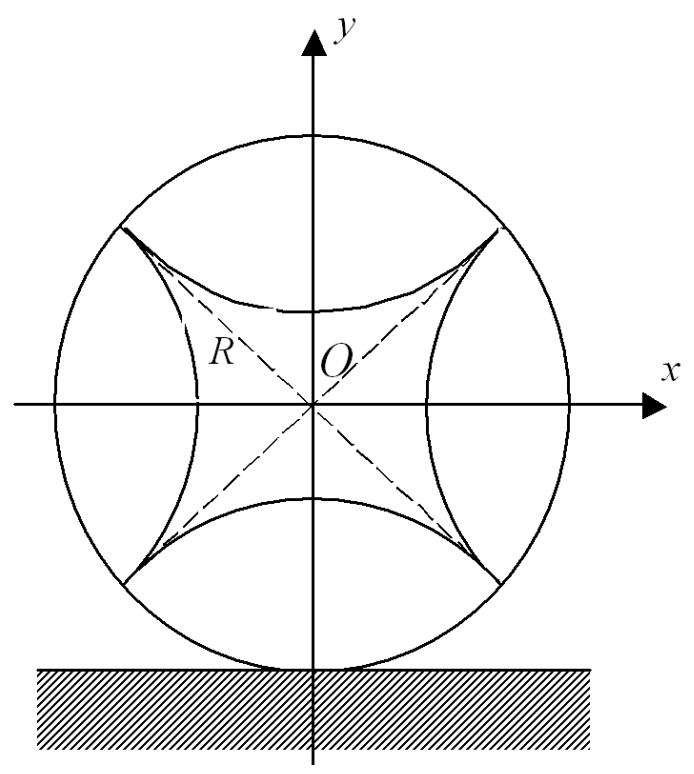

Figure 1: The omni wheel vertically aligned.

Note, in addition, that the curve of contacting points forms the $x y$-projection onto the wheel plane, having a shape of the circle of radius $R$, see Figure 1. Thus for translational and rotational motion we have continuity as well. Resúming we are able to conclude that the regularity of motion is conserved as roller switching at contact. At least on the level of integrity of the omni wheel. Recall, that all the description above takes place for vertical alignment of the omni wheel.

On the next level of assembling process, several omni wheels are interconnected with the moving platform of the vehicle, see Figure 2, using joint constraint as a class from the previous stage. In our case, number of wheels may be three or more. They can form different configurations on the platform body. We analyse an example with three wheels forming an equilateral triangle in the plane of the platform, see Figure 2, parallel to the coordinate horizontal plane $z x$. Axis $y$ here is assumed vertical.

\section{The roller dynamics model}

Firstly, we presume that the roller is axisymmetric spindle-shaped rigid body having outer surface defined in body frame of reference $O x y z$, see Figure 3, by

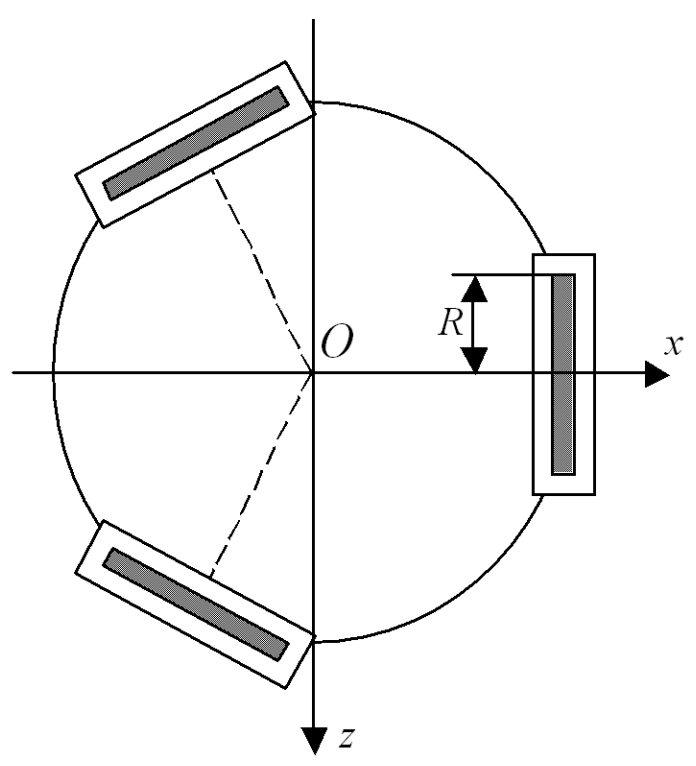

Figure 2: The three wheeled vehicle. Top view.

equation

$$
x^{2}+\left(\sqrt{y^{2}+z^{2}}+R_{1}\right)^{2}=R
$$

where $R$ is the omni wheel radius, $R_{1}=R \cos \alpha$ is the distance from the roller center to the wheel center, $\alpha=\pi / n$ is the half of the roller opening angle of visibility from the wheel center, $n$ is number of rollers per wheel.

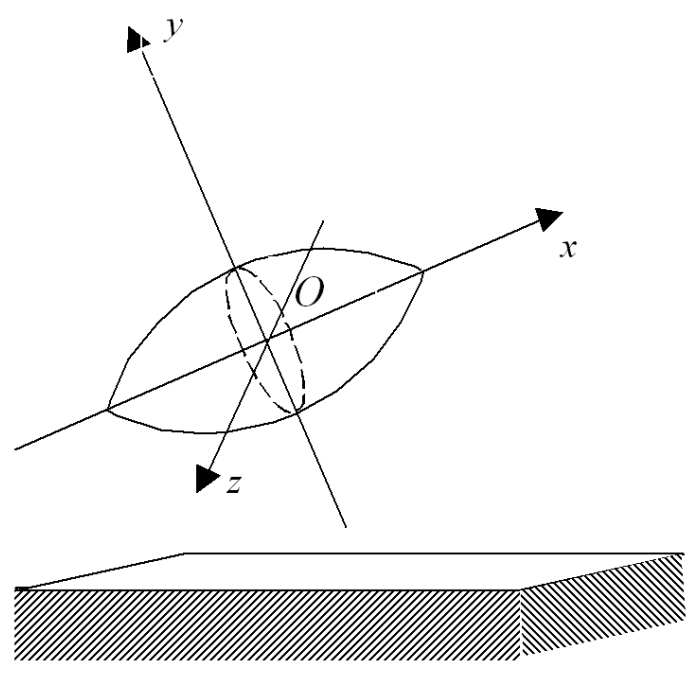

Figure 3: The roller over horizontal surface. Lateral view.

Dynamics of the roller translatory-rotary motion is implemented using equations of Newton - Euler as was shown in [6]. And rotational motion was modeled by the quaternion algebra [7]. 
Algorithm for contact tracking plays an important role for the correct and efficient work of the computer model of the contacting process of the roller and the horizontal surface. For modeling and simulation of the rigid body dynamics with unilateral constraint we apply the technology described in [8]. So we could have used in the object of contact a system of well known algebraic or implicit differential-algebraic equations. However, these equations degenerate at the roller tips defined by equations $x= \pm R \sin \alpha$ in the roller coordinates, see Figure 1. Usually, such a degeneration causes an abnormal completion of the simulation process.

In our case of the spindle-shaped roller over the horizontal surface, arranging the contact tracking procedure turns out being sufficiently simple. So one can point out explicit procedure for computing the nearest point $P_{B}$ of the roller to the horizontal surface, see Figure 4. This surface has its own nearest point $P_{A}$ at contact. Evidently the point $P_{A}$ is a vertical projection of the point $P_{B}$ of the roller.

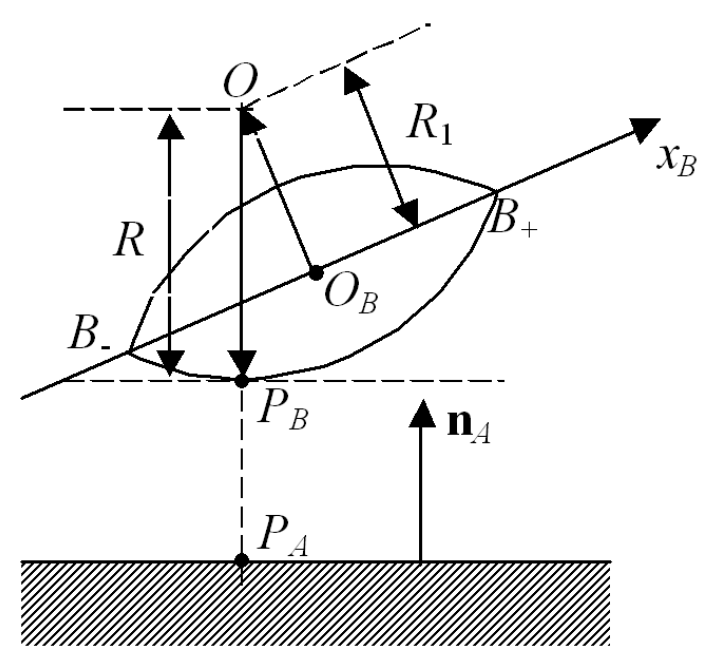

Figure 4: Contact tracking scheme.

Denote by $\mathbf{i}=(1,0,0)^{T}$ the unit vector along the axis $O_{B} x_{B}$ of the roller connected coordinate system from Figure 4. This vector is resolved with respect to (w. r. t.) the system $O_{B} x_{B} y_{B} z_{B}$. Let $T_{B}$ be the rotational matrix of the roller w. r. t. the inertial frame of reference. The latter frame, in our case, coincides with the fixed horizontal surface coordinate system $O_{A} x_{A} y_{A} z_{A}$. Also, let $\mathbf{r}_{B}$ be the roller current mass center radius vector w. r. t. the inertial system, and $\mathbf{n}_{A}=(0,1,0)^{T}$ be the ascending vertical unit vector. Simultaneously $\mathbf{n}_{A}$ is the normal vector to the horizontal plane.

Conventionally, we denote the plane as body $A$, and roller as body $B$. Let $\mathbf{d}$ be the horizontal unit vector defined by equation

$$
\mathbf{d}=\frac{T_{B} \mathbf{i}_{B} \times \mathbf{n}_{A}}{\left|T_{B} \mathbf{i}_{B} \times \mathbf{n}_{A}\right|} .
$$

Therefore, the directed segment $\overrightarrow{O_{B} O}$ must have a length $R_{1}$ and be defined by formula

$$
\overrightarrow{O_{B} O}=R_{1} \mathbf{d} \times T_{B} \mathbf{i}_{B}
$$

Here, $O$ is the curvature center for the circle of the roller vertical section, see Figure 4. This segment is located simultaneously in the vertical plane and in the wheel plane. Thus from Figure 4 we see that the lowest point $P_{B}$ of the roller outer surface is defined by equation

$$
\mathbf{r}_{P_{B}}=\mathbf{r}_{B}+R_{1} \mathbf{d} \times T_{B} \mathbf{i}_{B}-R \mathbf{n}_{A}
$$

since the $P_{B}$ lies on the same vertical with the point $O$ and on the circle mentioned above. To compute position of the point $P_{A}$ one has to put

$$
\mathbf{r}_{P_{A}}=\left(x_{P_{B}}, 0, z_{P_{B}}\right)^{T} .
$$

All the procedure above is valid only if the vector $T_{B} \mathbf{i}_{B}$ is inclined to the horizontal plane within an angle $\pm \alpha$. Otherwise one has to put $P_{B}=B_{-}$where $B_{-}$is the "left", see Figure 4, tip of the roller for angle of the vector $T_{B} \mathbf{i}_{B}$ inclination greater than the value $\alpha$. If this angle is less than $-\alpha$ then one has to guess $P_{B}=B_{+}$ where $B_{+}$is the "right" tip of the roller.

Finally, one can write down a contacting condition between roller and horizontal surface in the form

$$
\left|T_{B} \mathbf{i}_{B} \cdot \mathbf{n}_{A}\right| \leq \sin \alpha .
$$

This condition, however, is satisfied simultaneously for the lowest, being in contact, roller, and the highest one. To reject the latter case one can add to condition (4) yet another one

$$
y_{B}<R
$$

where $y_{B}$ is the altitude of the roller mass center w. r. t. inertial frame of reference.

So a conjunction of conditions (4) and (5) is equivalent to the case of contacting. Otherwise condition of normal reaction being zero should take place. Indeed, according to Signorini's law a following alternative is implemented for each individual roller: (a) contact takes place - relative normal velocity at contact should be zero; (b) contact is absent - normal reaction (and tangent too) of unilateral constraint should be zero. 
Condition (a) has several alternative possibilities of implementation. Firstly, from the geometric viewpoint a presence of contact is equivalent to the scalar condition

$$
y_{P_{B}}=0 .
$$

Its absence is equivalent also to the scalar condition

$$
F_{n}=0
$$

where $F_{n}$ is the normal component of a reaction force acting on the roller at the point $P_{B}$.

Computational experience show that equation of contact in the form (6) usually causes an abnormal termination of the simulation process for the dynamical model of the roller. One has similar result if we use equation

$$
v_{n}=0
$$

as an implementation of condition (a). Here $v_{n}$ is the normal component of the relative velocity at contact point. And only equation of the form

$$
\dot{v_{n}}=0
$$

leads to the required result: object of contact works properly during the simulation process. One has to recall here that all the implementation of the contacting process has the "rigid" point-contact model.

For each roller of the omni vehicle model when contacting the friction model being used is "turned on". In our model being developed the "simple" law of the Amontons - Coulomb dry friction is applied. Actually we use known piecewise approximation [8] to exact dry friction instead. This approximation has high accuracy over long time intervals [9]. In general, implementation of unilateral constraint model is based on the results outlined in [8].

If the angle of inclination for the roller axis of symmetry to the wheel plane has non-zero value then some of the above relations ought to be slightly corrected. In this case, rollers become distorted along the wheel rim. Given the position $\mathbf{r}_{O} \in \mathbf{R}^{3}$ of the wheel center, point $O$, see Figure 4, firstly, we have to build up an auxiliary base consisting of unit vectors:

$$
\mathbf{i}^{\prime}=T_{B}\left(\begin{array}{l}
1 \\
0 \\
0
\end{array}\right), \quad \mathbf{j}^{\prime}=\frac{\mathbf{r}_{O}-\mathbf{r}_{O_{B}}}{\left|\mathbf{r}_{O}-\mathbf{r}_{O_{B}}\right|}, \quad \mathbf{k}^{\prime}=\mathbf{i}^{\prime} \times \mathbf{j}^{\prime} .
$$

After that a matrix of coordinates change has the form $T^{\prime}=\left(\mathbf{i}^{\prime} \mathbf{j}^{\prime} \mathbf{k}^{\prime}\right)$ where $\mathbf{i}^{\prime}, \mathbf{j}^{\prime}, \mathbf{k}^{\prime}$ are assumed as vector columns. This matrix defines transformation from inertial frame of reference connected with the fixed body $A$ to the frame defined by the vector base $B^{\prime}=$ $\left\{\mathbf{i}^{\prime}, \mathbf{j}^{\prime}, \mathbf{k}^{\prime}\right\}$ introduced above in the following way

$$
\left(\begin{array}{l}
x_{A} \\
y_{A} \\
z_{A}
\end{array}\right)=T^{\prime}\left(\begin{array}{l}
x^{\prime} \\
y^{\prime} \\
z^{\prime}
\end{array}\right) .
$$

To reduce an analysis to the case of $\beta=0$ already considered above we have to rotate the base $B^{\prime}$ about $\mathbf{j}^{\prime}$ by the angle $-\beta$ such that after the rotation a new base $B=\{\mathbf{i}, \mathbf{j}, \mathbf{k}\}$ should be aligned with the wheel plane containing the unit vectors $\mathbf{i}, \mathbf{j}$. The rotation mentioned has the matrix

$$
S=\left(\begin{array}{ccc}
\cos \beta & 0 & -\sin \beta \\
0 & 1 & 0 \\
\sin \beta & 0 & \cos \beta
\end{array}\right)
$$

in the base $B^{\prime}$. Then in the base of the indicated body $A$ the rotation of the unit vector $\mathbf{i}^{\prime}$ can be represented as follows $\mathbf{i}=T^{\prime} S(1,0,0)^{T}$. Suppose also $\mathbf{j}=\mathbf{j}^{\prime}, \mathbf{k}=\mathbf{i} \times \mathbf{j}$. Evidently $\mathbf{k}=\mathbf{d}$ where $\mathbf{d}$ is the unit vector given above.

Thus based on the formula (2) and taking into account Figure 4 we can conclude that for the case of $\beta \neq 0$ the following result takes place

$$
\mathbf{r}_{P_{B}}=\mathbf{r}_{B}+R_{1} \mathbf{j}-R \mathbf{n}_{A}-\frac{R_{1} \tan \beta \sin \gamma}{\sqrt{1-\sin ^{2} \gamma}} \mathbf{j} \times \mathbf{i},
$$

where the angle $\gamma$ satisfies the equation

$$
\sin \gamma=\mathbf{i} \cdot \mathbf{n}_{A} .
$$

\section{Assembling vehicle model}

An assembling process of the omni vehicle prototype is implemented in two steps: (a) assembling the omni wheel consisting of the wheel itself and a set of rollers attached to the wheel; (b) assembling the vehicle by instantiating objects of the omni wheel class from stage (a) into the container class of the vehicle prototype.

To connect rollers, rather objects of the roller class, and the wheel we use model of the joint constraint previously developed and described in [5]. It is simply revolute class with free relative rotation about its axis. Codes of all the classes / models for the prototype are implemented as Modelica classes library. See visual model of the omni wheel in Figure 5. Here, in our example we selected for simplicity and certainty $n=4$.

The model of main interest is one of the whole vehicle which is "assembled" on the second stage of the assembling process. Connecting devices were also implemented as objects of the same joint class from stage 


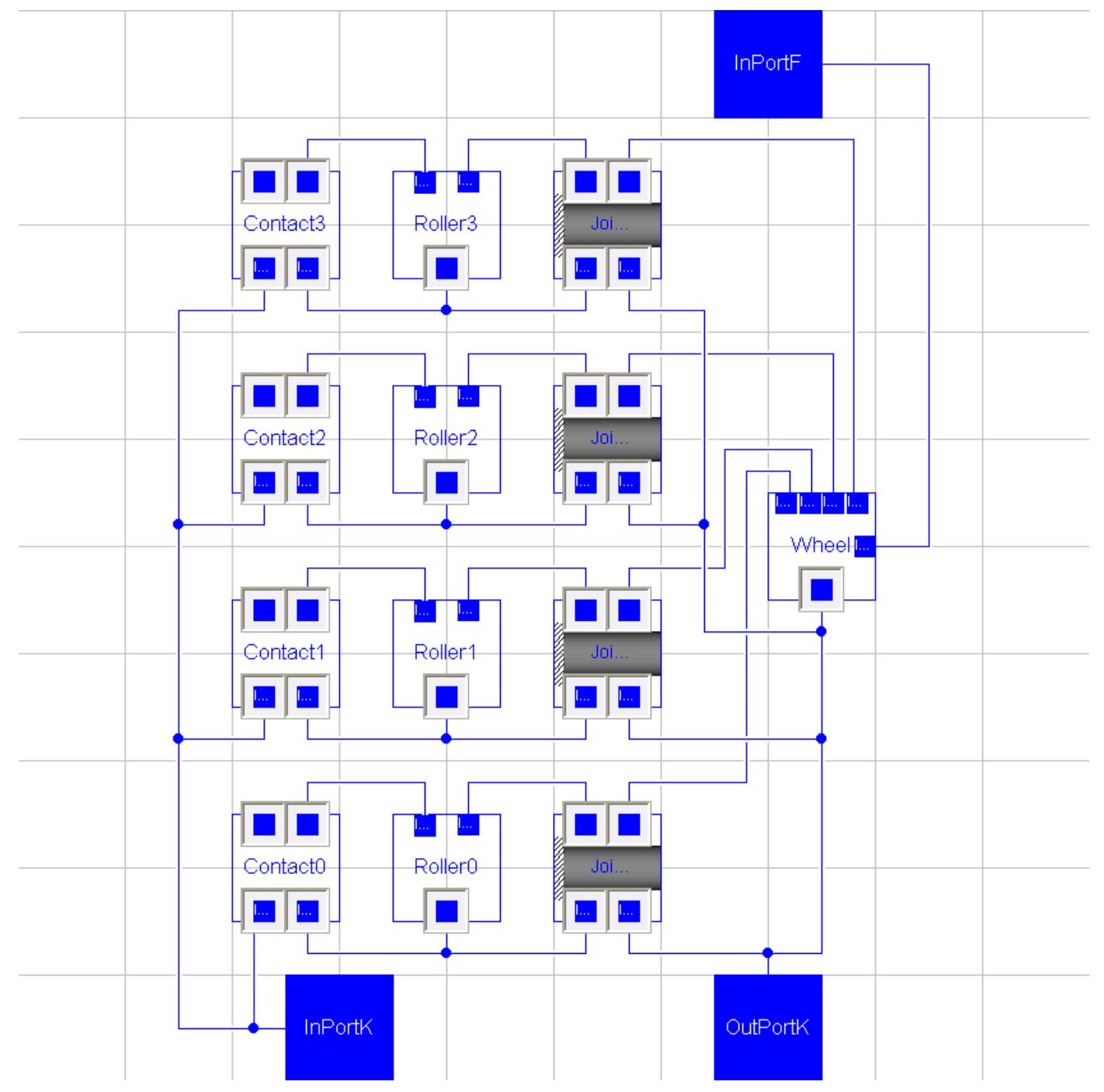

Figure 5: The omni wheel visual model.

(a). These joints connect the vehicle body and each of wheels. All joints above allow relative rotation without any resistance and lock sliding along the joint axis. See visual model of the vehicle in Figure 6. Here, for presentability, objects are shown as scalar elements. Actually, one has to instantiate corresponding arrays of objects of classes "Roller" and "OmniWheel" for arbitrary $n$ and arbitrary number of wheels in the vehicle.

Recall that before the DAE index reduction process implemented in Dymola the whole vehicle model consists of: (a) one rigid body of the vehicle platform; plus (b) three rigid bodies of the vehicle wheels; plus (c) twelve rigid bodies of rollers located on the wheels. According, for instance, to [5] for each object of rigid bodies we implement six Newton's ODEs for the mass center motion plus seven Euler's ODEs for rotational motion about the mass center. For the latter case we have four Euler's kinematical equations for the rigid body quaternion plus three Euler's dynamical equations for the rigid body angular velocity. Totally, the whole vehicle model includes system of ODEs of order $16 \cdot 13=208$. Besides, constraint objects are able to generate additional differential equations.

Wheels being assembled into the vehicle will keep the vertical alignment unavoidably. For this reason the simplified contact tracking algorithm described above works properly.

Computer experiments were performed for different numbers of rollers per wheel and using several friction models at contact between roller and the horizontal surface. Corresponding results were compared. For instance an evolution of the contact process for one wheel of the three wheeled vehicle is shown in Figure 7. Paying attention to the Figure legend we are seeing variables with suffixes ".h" and respectively curves of four colours. This variables represent so called mutual approaches for contacting bodies. Their values are simply distances between rollers of 


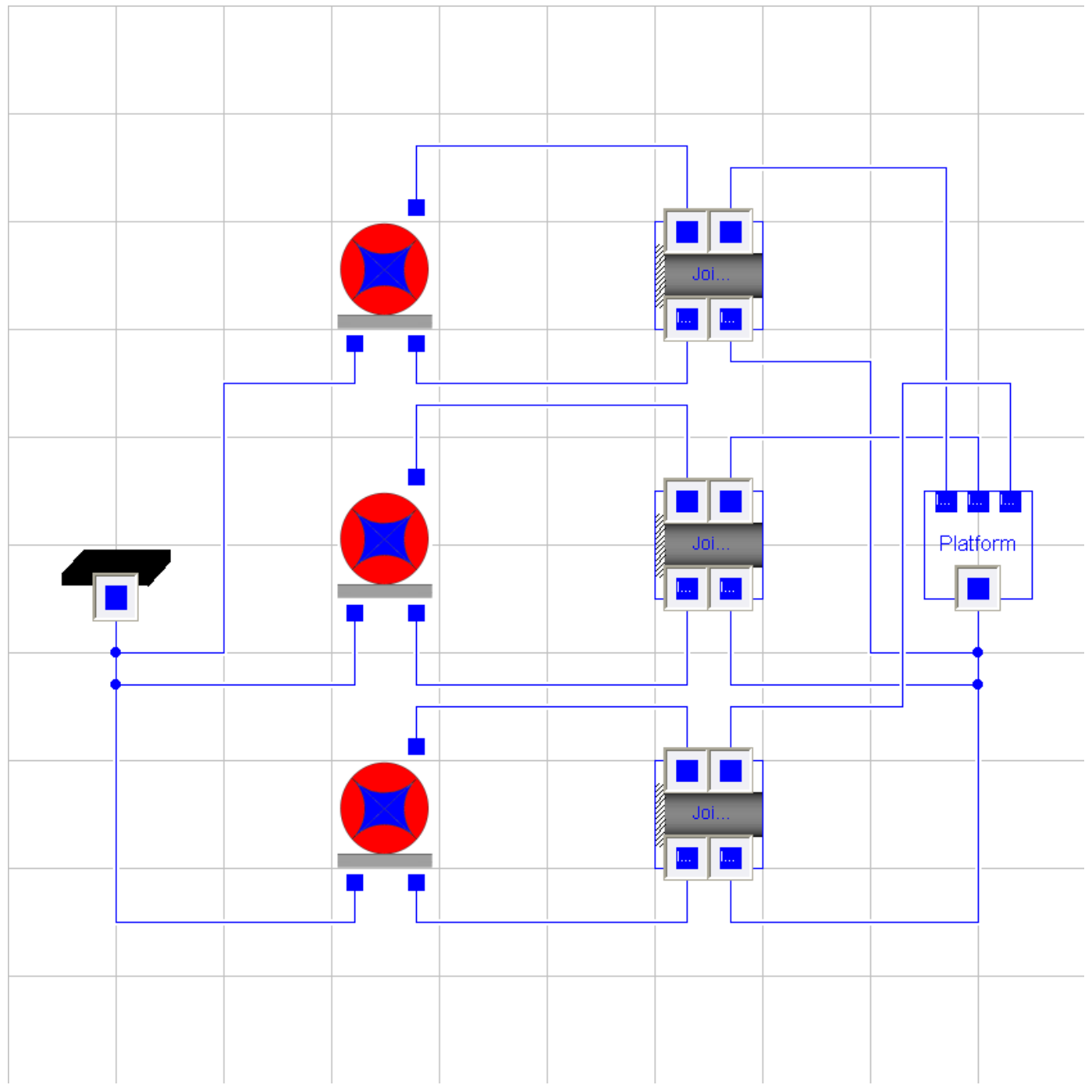

Figure 6: The omni vehicle visual model.

the wheel and the horizontal surface of rolling. These curves correspond to rollers being in different phases of wheel rotation: before contact, at contact, after the contact. See an instance of the roller change being zoomed in Figure 7. For implementing such a switching it is sufficiently simple to use if-clause thus alternating states of the contact existence / non-existence. Corresponding fragment of Modelica code may have the following representation ..

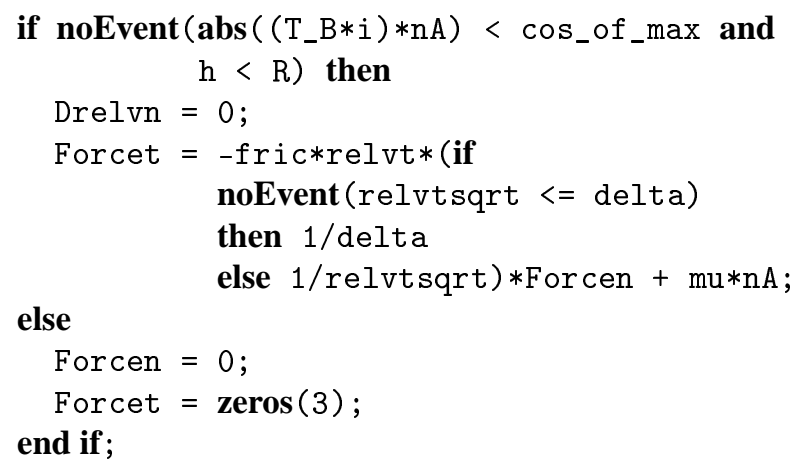

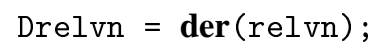

The first if-operator here is responsible for the unilateral constraint detection. Its condition is equivalent to conjunction of conditions (4) and (5). Drelvn is the variable being equal to the derivative of the relative normal velocity at contact. So we have an alternative: (a) Drelvn $=0$ means the contact existence or (b) Forcen $=0$ means contact absence or, equivalently, zero-valued force of reaction. Vector variable Forcet simulates tangent force of friction, being computed here using piece-wise linear approximation of dry friction.

Simultaneously, one can also observe the unilateral constraint accuracy being kept by the model at contacting, see Figure 8. In this Figure we can observe how a numeric error of the unilateral constraint feasibility slowly diverges, mutual approach ....h gradually 


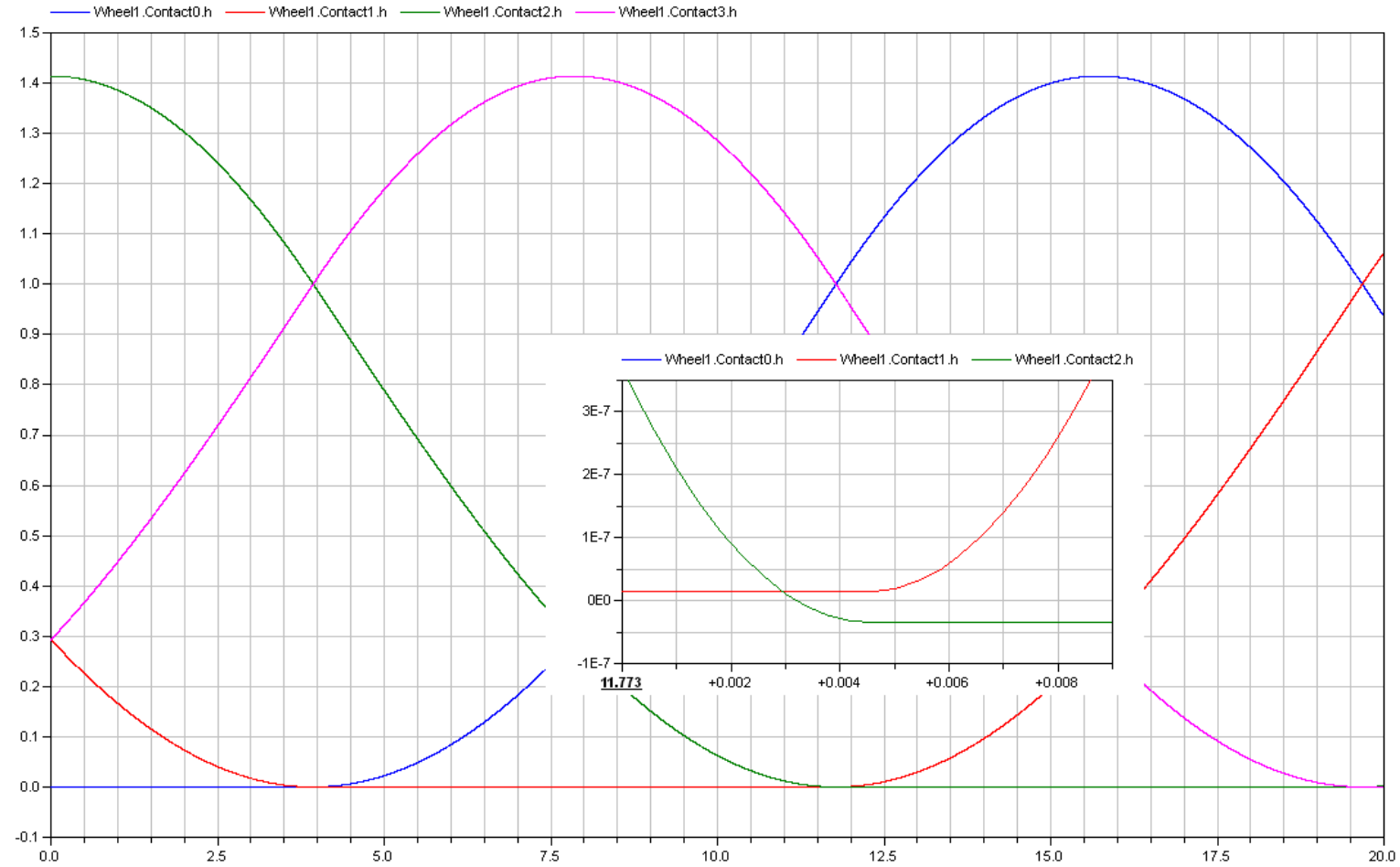

Figure 7: Process of rollers contact replacement

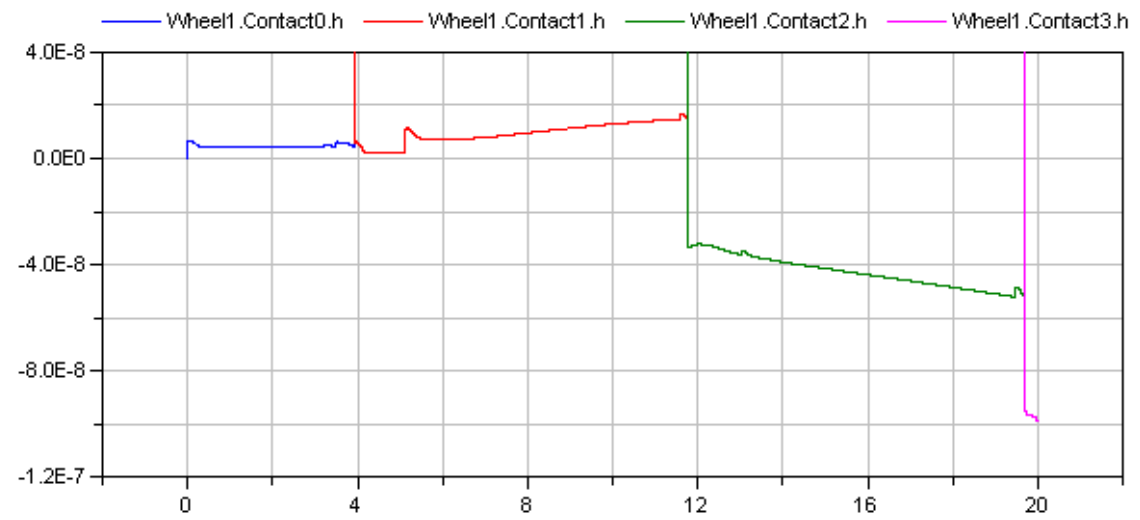

Figure 8: Accuracy of the unilateral constraint.

grows, for each successive roller in contact. Meanwhile, an absolute value of error stays near negligible value of $10^{-7}$ meters. Change of the curve colour corresponds to change of the contacting roller.

\section{Conclusions}

As a summary of main results obtained in the course of the omni vehicle model development we can highlight the following issues:

- There exist a possibility for smooth impactless switching between rollers at contact upon rolling of omni wheel;

- Efficient and simplified contact tracking algorithm was implemented;
- Dynamics of vehicle was investigated for different number of rollers per wheel;

- Influence of friction model on dynamics of the omni vehicle was analyzed.

This work was performed with partial support of RFBR, projects 11-01-00354-a, 12-01-00536-a, 1208-00637-a.

\section{References}

[1] Campion, G.; Bastin, G.; d'Andréa-Novel, B.: Structural Properties and Classification of Kinematic and Dynamic Models of Wheeled Mobile Robots. IEEE Transactions on Robotics and Automation, Vol. 12, No. 1, pp. 47-62, 1996. 
[2] Kálmán V.: Controlled Braking for Omnidirectional Wheels. International Journal of Control Science and Engineering, Vol. 3, No. 2, pp. 4857, 2013.

[3] Tobolár, J.; Herrmann, F.; Bünte T.: Objectoriented modelling and control of vehicles with omni-directional wheels. Computational Mechanics 2009. Hrad Nectiny, Czech Republic, November 9-11, 2009.

[4] Zobova, A. A.; Tatarinov, Ya. V.: The Dynamics of an Omni-Mobile Vehicle. Journal of Applied Mathematics and Mechanics, Vol. 73, Iss. 1, pp. 8-15, 2009.

[5] Kosenko I. I., Loginova M. S., Obraztsov Ya. P., Stavrovskaya M. S., Multibody Systems Dynamics: Modelica Implementation and Bond Graph Representation. In: Proceedings of the 5th International Modelica Conference, arsenal research, Vienna, Austria, September 4-5, 2006, pp. 213223.

[6] Kossenko I. I., Stavrovskaia M. S., How One Can Simulate Dynamics of Rolling Bodies via Dymola: Approach to Model Multibody System Dynamics Using Modelica. In: Proceedings of the 3rd International Modelica Conference, Linkopings universitet, Linköping, Sweden, November 3-4, 2003, pp. 299-309.

[7] Kosenko, I. I., Integration of the Equations of the Rotational Motion of a Rigid Body in the Quaternion Algebra. The Euler Case. Journal of Applied Mathematics and Mechanics, 1998, Vol. 62, Iss. 2, pp. 193-200.

[8] Kossenko I. I., Implementation of Unilateral Multibody Dynamics on Modelica. In: Proceedings of the 4th International Modelica Conference, Hamburg university of technology, Hamburg-Harburg, Germany, March 7-8, 2005, pp. 13-23.

[9] Novozhilov, I. V., Fractional Analysis : Methods of Motion Decomposition. Boston: Birkhauser, 1997. 